\title{
LA FRATERnitì TRA GAYA E ZAMbrano: AUTENTICITÀ NELLA CREAZIONE
}

ELENA LAURENZI

Università del Salento

\section{RESUMEN:}

La amistad entre María Zambrano y Ramón Gaya, que tiene sus raíces en el compromiso político de los años de la República y la Guerra Civil, maduró y se consolidó en los años del exilio romano en el terreno de la creación. En este ensayo me propongo analizar las concordancias existentes en sus respectivas concepciones de la actividad creativa. Para iluminar estas coincidencias, me refiero a la categoría de autenticidad de Ortega, mostrando cómo Gaya y Zambrano la traducen en los términos de la creación, pero también cómo profundizan y radicalizan su interpretación a través de las lecturas de Friedrich Nietzsche y Simone Weil.

PALABRAS CLAVE:

Creación, autenticidad, atención, Gaya, Zambrano, Weil.

\begin{abstract}
:
The friendship between María Zambrano and Ramón Gaya has its roots in the political commitment of the years of the Republic and the Civil War, but matured and consolidated in the years of their exile in Rome, on the terrain of creation. In this essay I propose to analyze the concordances existing in their respective conceptions of creative activity. To illuminate these coincidences, I refer to the Orteghean category of authenticity, showing how Gaya and Zambrano translate it into in terms of creation, but also how they deepen and radicalize its interpretation through the readings of Friedrich Nietzsche and Simone Weil.
\end{abstract}

\section{KEY WORDS:}

Creation, authenticity, attention, Gaya, Zambrano, Weil.

\section{Una fraternità all'insegna della creazione}

Una relazione di fraterna amicizia legò Ramón Gaya e María Zambrano, sigillata dall'appellativo semi-scherzoso con cui reiteratamente Gaya si rivolge alle sorelle 
Zambrano come «fratelle» e a María in particolare come «fratella massima» ${ }^{1}$. Le radici di questo rapporto privilegiato affondano nelle note vicende storiche che sedimentarono in un terreno di esperienze condivise, e che furono decisive per il consolidarsi del loro rapporto sul piano politico e su quello esistenziale: l'adesione alla Repubblica e il coinvolgimento nelle Misiones Pedagógicas, esperienza fondante per molti giovani di quella che Zambrano chiamava la «generación del toro» (Zambrano, 1995: 32); la partecipazione alla Guerra civile e la collaborazione in Hora de España, una rivista di poesia e letteratura «nacida en la guerra [que] no era de guerra $»^{2}$ (Zambrano, 1998; Trapiello 2011; Laurenzi 2016); la condanna all'esilio convertita in esperienza radicale di solitudine e di conoscenza. Ma, al di là e al di sotto delle circostanze, una vena profonda alimenta la loro amicizia: si tratta di una «affinità elettiva», come l'ha definita Pedro Chacón, determinata da una «reconocida comunión de creencias» e una «concordancia emocional y afectiva» (2011: 40). María Zambrano la dichiara esplicitamente nella lettera che scrive a Gaya dopo aver letto El silencio del arte: «me estremecieron las concordancias y coincidencias casi de expresión total» (Zambrano-Gaya, 2018: 42). L'affinità anche ideale e filosofica, che nasce negli anni giovanili dalla condivisione dell'impegno politico, matura e si consolida, negli anni dell'esilio romano sul terreno della creazione. In questo mio saggio, mi propongo di analizzare le concordanze esistenti non solo nelle rispettive concezioni dell'arte e della scrittura, ma più profondamente, nella loro pratica e nella loro esperienza dell'attività creatrice.

Come si sa, per Gaya la creazione si riferisce a un livello profondo dell'essere e dell'esistenza, che trascende l'arte, la tecnica, la produzione stessa dell'opera:

El arte, en contraste con la creación, es siempre una petulancia, un propósito, un lucimiento, un mérito, una... idea. La creación en cambio es [...] un acto vivo, un acto-naturaleza. La creación es un poder -el más grande, el más alto-, pero es un poder humilde; el verdadero creador está sometido a ese poder suyo, y cumplirlo no puede ser nunca un alarde (Gaya, 2010: 40).

A mio avviso, è proprio a questa dimensione -riferita all' «índole central, medular, inicial, original, del misterio creador» (Gaya, 2010: 103)- che Gaya allude quando

\footnotetext{
${ }^{1}$ L'appellativo, in italiano, compare nella dedica al volume della prima edizione (1969) di Velázquez, pájaro solitario donato da Gaya a Zambrano e riprodotto nel volume della loro corrispondenza (Zambrano-Gaya, 2018: 204).

2 Le citazioni dagli scritti di Gaya e Zambrano, così come del resto degli autori citati, vengono riportate nelle loro lingue originali. A causa delle restrizioni dovute alla pandemia COVID 19 mi è stato impossibile consultare i volumi dell'Opera completa di Zambrano pubblicati da Galaxia Gutenberg, per cui cito dalle edizioni per me attualmente accessibili.
} 
fa riferimento a una «raza» di poeti, pensatori e artisti in cui include se stesso e Zambrano $^{3}$. Per comprendere tale dichiarazione di comune appartenenza non basta richiamare l'apprezzamento mutuo e spesso entusiasta che pittore e filosofa professavano per i rispettivi lavori. Sul piano dei contenuti e dello stile, in effetti, non mancano di manifestare seri dissensi. Basti pensare alla discrepanza, anche aspra, nei giudizi, come quando Gaya si irrita per la passione di Zambrano nei confronti della pittura di Luis Fernández o di Juan Soriano (Gaya, 2016: 460, 417) o come quando Zambrano lamenta lo scarso interesse di Gaya per la pittura di Zurbarán (ZambranoGaya, 2018: 297). E il dissenso tocca anche questioni di sostanza, come quando Gaya critica l'eccesso di simbolismo nella scrittura di Zambrano (Gaya, 2010: 468) o Zambrano disapprova il procedere di Gaya per contrapposizioni ed esclusioni, nei suoi saggi sulla pittura (Zambrano-Gaya, 2018: 207). Tuttavia, queste dissonanze non impediscono che essi si incontrino a un livello più profondo, dove riconoscono la vocazione ineludibile -quasi un comandamento- che li spinge a creare, e che può essere anche «cruz» (Zambrano, 1987: 70) o «tortura» (Gaya, 2010: 68), ma dà senso alla loro vita.

«El arte es Destino -scrive Gaya- [...] no lo podemos construir nosotros, ni siquiera hacer nosotros, sino escucharlo y cumplirlo» (Gaya, 2010: 68). Nei suoi scritti autobiografici, anche Zambrano fornisce passi illuminanti sulla vocazione destinatale che in qualche modo dirige i suoi passi indipendentemente dal proposito -mai formulato come tale- di essere «un autor», e men che meno, un filosofo (Zambrano, 1971: 10). Nell'intervista rilasciata pochi mesi prima di morire, ripercorrendo le inquietudini manifestate fin da bambina nei confronti della filosofia, conclude: «Entonces no tengo más remedio que aceptar que mi verdadera condición, es decir, vocación ha sido la de ser, no la de ser algo, sino la de pensar [...] la de tener la paciencia sin límites que aún me dura para vivir pensando» (Zambrano, 1987: 71).

Negli interminabili anni dell'esilio la presenza dei divini demoni della creazione li ricompensa della vita che è stata loro sottratta, dando senso e pienezza alla loro esistenza ed elevandola a una dimensione impersonale. A questo fa riferimento Zambrano, nella lettera che scrive all'amico dall'Avana il 13 giugno del 1949, dopo aver ricevuto la notizia della morte di Fe Sanz, sotto le bombe: «Pero mira: tenemos nuestros Dioses y si sabemos hablarles y escucharlos, las cosas se hacen ellas solas [...]. Éntrate donde están tus Dioses y habla con ellos que será hablar contigo, y entonces verás muy claro» (Zambrano-Gaya, 2018: 26). E Gaya risponde, a stretto giro: «Si no fuera por esos dioses interiores, ¿dónde estaríamos

\footnotetext{
${ }^{3}$ Tra gli altri appartenenti a questa 'razza', Gaya cita Rafael Dieste, Cristobal Hall, Rosa Chacel, Salvador Moreno, Antonio Sánchez Barbudo, Concha Albornoz, Juan Gil-Albert, Soledad Martínez o Tomás Segovia.
} 
todos ya? En una sola cosa me siento cada vez más fuerte: mi pintura. Y cada vez me siento más comprometido. Es una alegría sentir que no somos libres. Sí, gracias a Dios, no tenemos esta monstruosidad vacía que se llama libertad» (ZambranoGaya, 2018: 28).

Al «talento endemoniado» di Zambrano (Gaya, 2016: 490), Gaya dedica un articolo pieno di ammirazione, pubblicato in ABC nel 1989:

Lo que sucede es que María, nuestra amiga, no se ha... puesto nunca a pensar como tantos -incluso algunas veces el propio Ortega- sino que ha pensado siempre como sin proponérselo, como sin quererlo, como sin... saberlo. Es también la manera de ser, de ser naturaleza que habíamos visto en Nietzsche. Estas buenas y extrañas personas -creadoras naturales de pensamiento, de poesía, de pintura, de música- más que hacer tal o cual cosa, parecen serla sin más... María Zambrano es, pues, una de esas criaturas... creadoras (Gaya, 2010: 873).

L'enfasi sulla vocazione 'demoniaca' non significa però affatto la celebrazione del genio inteso come spontaneità e ispirazione. Entrambi conoscono, al contrario, la pratica umile dell'artigiano, la regolarità del lavoro quotidiano, l'esercizio paziente e gratuito anche in assenza di un progetto chiaramente delineato, la meditazione prolungata e la contemplazione ricettiva e attenta. Entrambi sembrano far proprio il motto attribuito a Michelangelo Buonarroti secondo cui il genio è 'eterna pazienza'.

Nelle lettere spedite da Roma o da Parigi ai suoi giovani amici artisti -il poeta Tomás Segovia, il musicista Salvador Moreno-, Gaya insiste, con spirito pedagogico, sull'importanza della disciplina, del persistere nell'impegno assiduo e metodico anche quando non si ottiene il risultato. E a proposito dell'opera propria, racconta dei suoi innumerevoli «preparativos desesperantes o, mejor dicho, esperantes»: tentativi reiterati che giudica incompleti, non soddisfacenti, ma che rispetta e non rigetta, perché in essi riconosce la preparazione necessaria alla nascita di qualcosa di atteso, «algo decisivo que me alimente» (Gaya, 2016: 318). Così scrive a Moreno da Roma, nel marzo del '57: «He pintado (o, mejor dicho: trabajado) mucho, y creo que bien, o bien dirigido, pues todavía no veo surgir ese cuadro... libre de todo, despreciativo de todo, que yo espero de mí, aunque no guste a nadie» (Gaya, 2016: 508). In una lettera all'amica Teresa de la Serna scritta da Venezia il 31 gennaio 1953, troviamo una descrizione dettagliata di questa sua maniera di lavorare, tesa tra l'imperativo assoluto della vocazione e l'accettazione umile del risultato relativo, necessario non solo per motivi economici, ma come preparazione ed esercizio: 
He trabajado mucho, pero hasta ayer no he pintado algo que me parezca decisivo, pues ya sabes que doy muchas vueltas antes (cosa que no han comprendido nunca las gentes y siempre me han juzgado [...] por los frutos de estos rodeos, de eso que yo llamo ahora sitiar) así que tengo muchísimas cosas - que me servirán, creo, para sacar dinero para otra temporadita- y muy poco en realidad que pueda satisfacerme (Gaya, 2016: 324).

La pratica di «dar rodeos» e «sitiar» appartiene anche al processo creativo di María Zambrano. I materiali conservati nell'archivio della Fondazione a Vélez Málaga documentano il suo lavoro costante di scrittura e riscrittura, che prende forma in molteplici versioni dello stesso testo, spesso ricorretto persino nella copia pubblicata. Poiché la creazione è sempre trascendenza, un andare oltre, rispetto a cui l'arte è solo «un tránsito [...] un lugar de paso» (Gaya, 2010: 77). E tuttavia, è un transito necessario, imprescindibile, perché la vocazione possa essere verificata, compresa nel suo significato più profondo. Nei confronti dell'opera si dà dunque, a un tempo, identificazione e disidentificazione: «Tengo varios libros escritos, ninguno de ellos me gusta - afferma Zambrano- y es que siento que no es mío, que es más que yo» (Zambrano, 1987: 70). E Gaya riflette su questo paradosso della necessità dell'opera e della sua eccedenza:

[...] hay que hacer a toda costa. Lo que importa es, naturalmente, ser, pero no se es si no se ha hecho, aunque esto que se hace, y que produce el ser, no es, en cambio, lo que vale, al final [...] la obra no es nada, en efecto, pero es ineludible, no se puede prescindir de ella para pasar a ser, ya que ser verdaderamente es un encuentro con algo que ya somos y algo que tenemos que lograr ser (no lograr hacer), y ese encuentro es lo que sucede únicamente después de la obra y, en absoluto, antes (Gaya, 2016: 21).

\section{Il principio di autenticità: da Ortega a Nietzsche e Weil}

L'insistenza da parte di Gaya e Zambrano sulla necessità di riconoscere o incontrare, nel terreno della creazione, qualcosa che si è tenuti a essere o diventare nella vita, permette di leggere il senso profondo della loro opera alla luce del concetto di autenticità.

Autenticità è una parola che Gaya usa per definire il proprio carattere, in particolare quando confessa la propria mancanza di diplomazia, la tendenza a dire le cose chiaramente a costo di urtare sensibilità e suscettibilità: «en esos momentos de... peligro social, yo me decido por la autenticidad», scrive a Salvador Moreno nel 1957 (Gaya, 2016: 504). Il termine non appare frequentemente nei suoi saggi di 
pittura. Tuttavia, nell'epistolario troviamo prove del fatto che considera l'autenticità un criterio pertinente nel giudizio su un artista o un'opera, e che può persino prevalere sullo scarso apprezzamento tecnico o propriamente artistico. Così, in riferimento al pittore asturiano Dario de Regoyos, scrive nel 1956 a Salvador Moreno: «me gusta -o me gustaba- (aunque yo no tenga nada en común con él), por su autenticidad, por el carácter cerril de su autenticidad» (Gaya, 2016: 469), anche se si tratta una autenticità «sin inspiración», a differenza della autenticità «fluida», «libre», che riconosce a pittori più ammirati: Murillo, Corot, Cezanne, Constable e-ovviamente-Velázquez. Ancora più rilevante è un passo di una lettera a Juan Bonafé, dove Gaya attribuisce la propria inattualità -nel senso nietzscheano del termine- all'effetto scomodo della propria pittura sulla sensibilità artistica corrente, poiché essa esige autenticità a un pubblico che è invece corrotto dall'artificiosità dell'arte e dall'intellettualismo della critica:

Estamos [...] en guerra con nuestra contemporaneidad [...] no caemos nosotros mismos en la cuenta de que la pintura que queremos hacer es hoy excesivamente revolucionaria, imposible de ver por parte de nuestros contemporáneos [...] volver a la razón, a la autenticidad, a la desnudez inicial y de valor permanente dentro del mundo loco y viciado de hoy tiene que resultar horrible (Gaya, 2016: 603).

Anche per Zambrano, l'autenticità è una condizione essenziale della persona e della libertà, (Zambrano, 1988; Laurenzi 2018), che non può non investire la sfera della creazione. Nel suo saggio inedito su Dante, scritto nel $1966^{4}$, si riferisce all'unione di mente e cuore che contraddistingue l'opera dantesca riconducendola alla virtù medievale della lealtà, e suggerisce che tale connubio tra sentire, pensare e operare («unidad de mente, ánima y acción»; Zambrano y Laurenzi, 2007: 64) potrebbe essere tradotto nel presente attraverso il concetto di autenticità. In contrapposizione alla virtù egocentrica della sincerità che è propria del solipsismo della coscienza moderna, la quale ha perso la solidarietà con il reale, il concetto orteghiano di autenticità sembrerebbe poter riconcettualizzare in termini ontologici, etici ed estetici, l'appartenenza dell'essere umano al mondo. Tuttavia, Zambrano chiarisce, in questo testo, che considera l'idea corrente di autenticità non abbastanza articolata e profonda. Per cercare di comprendere le implicazioni della sua concezione, risulta allora utile fare un breve richiamo a Ortega.

Ortega, come è noto, usa il termine autenticità nella sua riflessione sulla vita umana individuale intesa come vocazione e destino, e nella conseguente riformulazione dell'etica, non più concepita come accettazione e obbedienza a una

\footnotetext{
${ }^{4}$ Pubblicato in edizione bilingue in Zambrano e Laurenzi (2007).
} 
norma, ma secondo il mandato pindarico (già fatto proprio da Nietzsche) che chiede di essere ciò che si è (Ortega, 2006). In ottemperanza alla sua visione ontologica della unità composta dall'io e la propria circostanza, Ortega considera l'autenticità in due direzioni: nei confronti della circostanza, essa è imperativo di amore e di salvazione nell'orizzonte del senso; nei confronti dell'essere individuale, è impegno a realizzare la propria vocazione. Come ha sottolineato José Lasaga Medina, l'autenticità orteghiana riferita alla vocazione non va confusa con l'intimità del sentimento, secondo la visione romantica à la Rousseau. Ha piuttosto la consistenza del daimon socratico, di un progetto in divenire che il soggetto subisce prima ancora di realizzarlo:

«Vocación», por lo tanto, nombra el componente de reclamación, de exigencia, de misión en que tiene que consistir mi vida, su destino [...]. El término usado por Ortega tiene un matiz religioso que ayuda a perfilar su verdadero alcance, pues en la medida en que se trata de una «llamada», ésta no procede de la propia conciencia, aunque sea ahí donde se manifieste: es decir, que la vocación es heterónoma respecto de la voluntad y la razón humana (Lasaga Medina, 2006: 193).

Nella riflessione di Zambrano e di Gaya questa eteronomia o trascendenza dell'autenticità rispetto all'io e alla sua volontà è più marcata e profonda rispetto a quanto non lo sia per Ortega. Quest'ultimo, infatti, pensa l'autenticità nella cornice di una concezione sportiva e agonistica del rapporto uomo-mondo (Rockwell, 1994) e nell'ambiguo riferimento al progetto come espressione della vocazione (Laurenzi, 2018). Nei nostri due creatori, invece, si afferma con più nitidezza l'aspetto della passività, intesa sia come passione, e quasi possessione del daimon che spinge a creare, sia come attenzione e accoglienza nei confronti della realtà di cui è parte il creatore, e che è la premessa della creazione autentica. In questa prospettiva, l'idea orteghiana dell'autenticità viene profondamente rielaborata e anche trasfigurata. La mia ipotesi è che in questa rivisitazione giochino un ruolo ispiratore e illuminante due autori intensamente frequentati da entrambi: Friedrich Nietzsche e Simone Weil.

Sia per Gaya che per Zambrano, la relazione con Nietzsche è una sintonia tra spiriti liberi, solitari e inattuali. Il filosofo dello Zarathustra e dell' Aurora illumina le svolte cardinali della riflessione di Zambrano, laddove il magistero di Ortega non le fornisce strumenti adeguati (Laurenzi, 2012) e la lettura di Ecce Homo le consegna un'esperienza della vocazione più consonante alla propria: la narrazione della passione necessaria per diventare ciò che si è.

Anche Gaya, come ricorda Andrés Trapiello nel suo prologo alla Corrispondenza di Gaya, sente fin da giovane un'ammirazione viva per Nietzsche e la coltiva nella maturità, tanto che nel 1966, rispondendo a una inchiesta su quali considerasse le 
quindici grandi opere imprescindibili della letteratura universale, cita l'epistolario del filosofo tedesco accanto a l'Iliade e al poema Árboles hombres del suo ammirato Juan Ramón Jiménez. È altresì commovente la testimonianza dello stesso Trapiello circa una lettera autografa di Nietzsche che Gaya comprò nel suo primo viaggio a Parigi, e di cui non si disfece mai:

Fue una de las contadas pertenencias que lo acompañaron por donde anduvo. Se diría que la necesitaba como recordatorio y sostén [...] la tenía siempre a la vista No colgada en la pared, como podría acaso exhibirse en un museo o entre los trofeos de un coleccionista, sino sobre una mesita, en una estantería, en una alacena, con su marquito de madera, cambiando de lugar y algo apartada, en verdad como algo vivo [...] y no muy lejos de una fotografía del propio filósofo recortada de una revista. No había en su casa una presencia tan visible de nadie, si exceptuamos los pequeños «acentos» aquí y allá, entre sus libros y objetos, de Juan Ramón Jiménez o de Victoria de los ángeles... Se diría que la foto y la carta de Nietzsche, así como estas otras pocas «memorias» de algunos amigos escogidos, su verdadera familia, le recordaban quién era y quién quería ser (Trapiello, en Gaya, 2016: 12).

Allo stesso modo, Simone Weil rappresenta per il pittore un'autrice di riferimento e persino un 'feticcio': anche di lei conserva la foto nella sua collezione di immagini (un «fondo escogido y riguroso»; Gaya, 2016: 434). E come María Zambrano, che meditava di tradurne in spagnolo una scelta di saggi (Campo, 2009) -anche Gaya a un certo momento della sua vita è tentato di creare qualcosa ispirandosi alla grande pensatrice francese-. Scrive in questo senso, nel 1952, a Salvador Moreno:

Manolo Durán me dio la fotografía que me enviste de Simone Weil que como puedes imaginar te agradezco mucho (ya está clavada en mi cuarto de Cuernavaca junto a Pastora y a la crucecita que pintaste); la mirada es estupenda, claro, y desde luego no la encuentro nada fea [...]. Por cierto, que empecé estos días una cosa sobre ella o mejor sobre algunas de sus iluminaciones geniales (Gaya, 2016: 222).

La figura della filosofa e miliziana anarco-sindacalista era loro probabilmente già nota negli anni della Guerra civile. Ma negli anni romani, entrambi hanno modo di approfondire la conoscenza delle sue opere anche grazie alla comune amicizia con la scrittrice Cristina Campo, appassionata studiosa e traduttrice della Weil in Italia. E proprio Campo, in una lettera al traduttore Leone Traverso, propone un interessante parallelismo tra Gaya e Weil alla luce dell'etica dell'autenticità nella creazione e dell'intolleranza nei confronti dello specialismo e l'elitismo degli intellettuali professionisti. Per illuminare i tratti comuni tra i due, fa riferimento alla 
La Fraternità tra Gaya e Zambrano: autenticità nella creazione

figura dell'idiota del villaggio, ricorrente in Weil (e ripreso, come si sa, anche da Zambrano, 2019).

Gaya -commenta Campo- è della stessa razza [di Weil]. Dice cose ancora più estreme, se è possibile. È veramente il pazzo del villaggio, il nano di Velzquez o di Shakespeare, a cui solo è concesso di dire la verità (Campo, 2007: 108).

\section{La contemplazione attenta e misericordiosa}

Tra le suggestioni importanti del pensiero di Weil che riverberano nella concezione creatrice di Zambrano e di Gaya, c'è il concetto di attenzione. Per Weil questo è il principio focale tanto dell'etica come dell'estetica:

Le poète produit le beau par l'attention fixée sur du réel. De même l'acte d'amour. Savoir que cet homme, qui a faim et soif, existe vraiment autant que moi -cela suffit, le reste suit de lui-même. Les valeurs authentiques et pures de vrai, de beau et de bien dans l'activité d'un être humain se produisent par un seul et même acte, une certaine application à l'objet de la plénitude de l'attention (Weil, 1947: 120).

L'attenzione weiliana rappresenta per Gaya e Zambrano un tema filosofico fecondo, a supporto della scelta del realismo contro il razionalismo imperante nella modernità occidentale, e contro ogni forma di arte o filosofia disumanizzata (dall'astrattismo allo strutturalismo). Il realismo che professano è d'altra parte distante dalla pedissequa e greve riproduzione di ciò che è (anche nella forma della denuncia propria dell'arte 'impegnata'), poiché concepisce piuttosto la realtà come deposito del sacro (Durante, in Zambrano-Gaya, 2018) e come mistero che trascende, la cui verità si rivela allo sguardo paziente dell'amore.

Osserva Pedro Chacón:

Ambos compartían una similar concepción respecto de la verdad de lo real, de aquello que se deja transparentar tras lo que se nos muestra, y ambos sintonizaban en la actitud que debían mantener su pensamiento y su pintura ante esa realidad: medios pasivos, obedientes y caritativos de su revelación. Cualquier acto creativo es, para María Zambrano y para Ramón Gaya, un acto desvelador del misterio que encierra la realidad, de lo sagrado escondido porque, tanto para uno como para el otro, la realidad es sagrada (Chacón, 2011: 51). 
L'accettazione piena della realtà senza belletto, senza moralismo, contemplata nei suoi abissi, deriva anche dalla lettura di Nietzsche, come Gaya riconosce esplicitamente:

Toda virtud, toda calidad, todo valor que no lleva en sì su propio abismo, su propio peligro, incluso su propio lado malo, no me parece algo respetable, completo; de ahí el asco (cada vez mayor) que siento por todos los puritanismos, las bondades, por las buenas cualidades, por los valores... si estos valores se presentan separados, abstraídos, hechos abstracción, sin vida. Todo esto, claro, no es nuevo, pues Nietzsche lo ha visto, o mejor, lo ha sentido mucho antes (Gaya, 2016: 496).

Nell'interpretazione dei nostri due autori, tuttavia, l'attenzione weiliana e il vitalismo nietzscheano si trovano declinati secondo la visione misericordiosa che è presente nella tradizione letteraria e pittorica spagnola. Galdós e Velázquez sono i loro modelli prescelti, autori caratterizzati da una creazione che si nutre dell'attenzione pietosa, a un tempo distaccata e partecipe, nei confronti del reale, e che proprio grazie a questa attenzione risultano capaci di portare alla trascendenza le vite minuscole dei personaggi. Nel suo omaggio a Galdós Zambrano, descrive, affascinata, il metodo di lavoro dello scrittore: la sua osservazione attenta e serena, partecipe e disincantata al contempo, e il suo ascolto benevolo, che gli permette di ritrarre la vita delle strade di Madrid in tutte le sue manifestazioni, sfumature, timbri, colori, dialetti. Analogamente Gaya insiste sulla contemplazione intrisa «de un piadoso amor impersonal» che Velázquez volge alla realtà: l'«atención humildísima» alla natura morta, l'«amoroso desapego» che traluce nei panorami, e soprattutto l'«entrañable proximidad» verso gli esseri umani. Grazie a questa sua prossimità «intensa y fraterna», le sue figure risultano liberate dall'impalcatura del personaggio così come dalla caratterizzazione della persona, per apparire come «seres desposeidos», «llevados a su anónimo rincón vital» (Gaya, 2010: 135; Morey, 2003). Perciò i suoi buffoni non sono grotteschi, fenomeni da baraccone, bensì creature, esseri che vengono accolti e restituiti nella loro unicità, come avviene con la figura della Maribárbola ne Las Meninas, che Gaya assimila a «una flor un tanto desproporcionada (a la manera, por ejemplo, de los girasoles), fuera de escala, contrahecha, pero viva, con la legitimidad de la vida y recibiendo muy confiadamente en el rostro la luz tierna, igualadora, del día velazqueño» (Gaya, 2010: 120).

Ma è soprattutto la contemplazione del Niño de Vallecas -un quadro molto amato anche da Zambrano (Chacón, 2015) - ad offrire a Gaya l'opportunità di descrivere -in modo magistrale- l'attenzione pura del creatore dai tratti nitidamente weiliani, che diventa celebrazione del mistero della realtà e della sua trascendenza: 
En ese rostro tierno, manso, santo, animado por una sutil mueca agridulce, es donde con más limpieza parece producirse el sacrificio de la realidad y también el sacrificio del arte [...] aquí pintura y realidad -sin ser alteradas ni evitadas- parecen trocarse, de pronto, con otra cosa, en algo como un cántico, no un cántico artístico, sino un cántico sagrado [...]. Ante El niño de Vallecas Velázquez no actúa en absoluto no se compadece, no se lamenta, no sufre ni se complace, no se burla o ensaña, ya que ha logrado por fin su más perfecta pasividad creadora; a El Niño de Vallecas Velázquez lo deja, intacto, vivir, venir a vivir, a estarse entero y verdadero en su gloria de ser vivo, dueño en rotundo de su ser central [...]. Ante esa extraña criatura de Dios, Velázquez permanecerá completamente inmóvil, tenso, sin decir nada, y dejará que hable la criatura misma, o mejor, su ser desnudo, su ser solo, libre, liberado, salvado de sí (Gaya, 2010: 120-121).

Al cospetto del reale, dunque, l'atteggiamento della filosofa e del pittore si mantiene ricettivo e passivo, «no pasa por una actitud avasalladora que intente captarla mediante una técnica, un estilo, un método, o apresarla en una red de ideas, sino sólo a través de un humilde apego y amor a la realidad misma» (Chacón, 2011: 49). In questo senso, Gaya arriva a teorizzare, come si è visto, la 'passività creatrice', in cui l'artista a poco a poco rinuncia alla sua «acalorada actividad artística» per fare spazio alla realtà «entregándose a una especie de mansedumbre creadora» (Gaya, 2010: 108). Questo atteggiamento che è proprio dell'autenticità nella creazione ha $\mathrm{i}$ tratti della obbedienza e dell'umiltà indicati da Weil:

Si on suspend le travail de l'imagination combleuse et qu'on fixe l'attention sur le rapport des choses, une nécessité apparaît à laquelle on ne peut pas ne pas obéir. Jusque-là, on n'a pas la notion de la nécessité ni le sentiment de l'obéissance. Alors on ne peut pas être orgueilleux de ce qu'on accomplit, quand même on accomplirait des merveilles (Weil, 1947: 55).

Chacón sottolinea molto giustamente come questa concezione della creazione supponga un coinvolgimento personale, un processo di profonda trasformazione e anche di purificazione da parte del creatore: «una purificación personal del propio creador que le posibilita establecer una relación de fusión con la realidad a la que cabe calificar de "mística", pues no se trata de "entenderla", sino de serla» (Chacón, 2011: 52). Possiamo leggere anche questa esigenza alla luce del concetto weiliano di decreazione. Così come, secondo la filosofa francese, la creazione del mondo è stata possibile grazie al ritirarsi di Dio per lasciare il posto all'apparizione del creato e alla sua trascendenza nella tensione amorosa ( «C'est Dieu qui par amour se retire de nous 
afin que nous puissions l'aimer. Car si nous étions exposés au rayonnement direct de son amour, sans la protection de l'espace, du temps et de la matière, nous serions évaporés comme l'eau au soleil»; Weil, 1947: 40), analogamente all'essere umano è richiesto un atteggiamento di rinuncia: una sottrazione dell'io, che -nel caso del creatore- implica la rinuncia all'ambizione e all'orgoglio dell'artista, ma anche alla presunzione del progetto o della tecnica, così come al proliferare dell'immaginazione «combleuse de vide» (Weil, 1947: 25). Solo facendo il vuoto, infatti, la realtà può penetrare nell'opera e nell'anima dello stesso creatore, operando in lui una sorta di metamorfosi: «el acto de creación -scrive Gaya- es un acto de metamórfosis, es decir, en que se encarna algo, al dejar, algo, de ser» (Gaya, 2016: 326). Cristina Campo coglie la matrice weiliana di tale concezione della creazione intesa come compenetrazione e intima comunione con il reale, e ne dà una lettura profondamente religiosa nel suo saggio su Gaya:

sicché l'arte stessa non è che un mezzo, una 'trasparenza' un ingiudicabile modo di colloquio tra Dio e Dio tramite un uomo che vuole solo non esserci. L'arte, scrive Gaya, non è come si è detto una corporeità ma una concavità... L'arte sembra giungere di molto lontano, passare attraverso l'uomo, indi sbarazzarsi dell'uomo come di una corteccia e proseguire. Di qui l'ingombro dello stile 'in cui l'arte si rifugia quando si travia', dando luogo al carattere, all'espressività, all'emprise della persona sulla realtà. Ma nell'artista puro la creazione è nulla più che obbedienza, risposta a quella realtà che vuol essere salvata, fatta trasparente attraverso di lui, attraverso cioè un'anima nuda (Campo, 2008: 15).

María Zambrano, nel suo scritto La pintura en Ramón Gaya, rimasto inedito e ora pubblicato nel volume della loro corrispondenza, sembra partire da queste considerazioni per fare un passo in più nella direzione dell'autenticità, suggerendo che, attraverso questa nudità dell'anima attenta, possa darsi una circolazione virtuosa tra l'opera e il fruitore: un «rispondersi di echi che si dà qualche volta tra l'arte e la contemplazione dell'arte», come scrive anche Campo. Nella pittura di Ramon Gaya -osserva Zambrano- «queda como invisible el pintor. Y más que el pintor mismo, ese "yo" que la mayor parte del arte contemporáneo nos arroja a la cara [...] estamos en la pura libertad, en esa que se gana a fuerza de humildad» (Zambrano-Gaya, 2018: 121, 128). Questa nudità, questa passività creatrice, questa contemplazione attenta che presuppone una metamorfosi, una purificazione del creatore, è a sua volta l'effetto che la sua arte produce su chi la contempla: «El espectador que ha sabido quedarse en su pasmo se siente poderosamente y sutilmente subyugado y atraído, llamado por algo que pide ser seguido: es la pintura en su paso que así lo mueve. Y, si la sigue, entra ya a contemplar» (Zambrano-Gaya, 2018: 123). In questo consiste 
-conclude la filosofa-la «virtud catártica y moral» dell'arte, l'«ética que se desprende de toda creación humana». La contemplazione che la pittura autentica esige, sollecita e finalmente provoca, «pone en ejercicio» in chi la osserva un'attitudine corrispondente di autenticità: una condizione o atto in cui si coniugano attenzione e passività - «la máxima vigilancia de la conciencia y la pasividad del alma que acoge la realidad sin recelo»- e che si crea nel «centro invulnerable» della persona (Zambrano-Gaya, 2018: 24): il luogo dove la solitudine si apre alla comunione.

\section{Riferimenti bibliografici}

Campo, Cristina, Caro Bul: lettere a Leone Traverso (1953- 1967), Milano, Adelphi, 2007.

Campo, Cristina, Se tu fossi qui. Lettere a María Zambrano 1961-1975, Milano, Archinto, 2009.

Campo, Cristina, Il sentimento della pittura di Ramón Gaya, in AA. VV., Ramón Gaya, Antologica 1948-1999, Murcia, Museo Ramón Gaya, 2008.

Chacón Fuertes, Pedro, «Ramón Gaya-María Zambrano: Elective Affinities», Escritura e imagen, Vol. 7, 2011, págs. 39-58.

Chacón Fuertes, Pedro, «La pintura como lugar de revelación en María Zambrano», Aurora: papeles del Seminario María Zambrano, n. ${ }^{\circ}$ 16, 2015, págs. 28-41.

Gaya, Ramón (2010), Obra completa, Valencia, Pre-Textos, 2010.

Gaya, Ramón, Cartas a sus amigos, Valencia, Pre-Textos, 2016.

Lasaga Medina, José, Figuras de la vida buena, Madrid, Enigma Editores, 2006.

Laurenzi, Elena, Sotto il segno dell'aurora. Studi su María Zambrano e Friedrich Nietzsche, Pisa, ETS, 2012.

Laurenzi, Elena, El testimonio de las luciérnagas. María Zambrano y la experiencia de la guerra de España, in Bea, E. y Fernández Ruiz-Gálvez, E., Cien años de discurso femenino sobre la guerra y la paz, Valencia, Tirant humanidades, 2016.

Laurenzi, Elena, Il paradosso della libertà. Una lettura politica di María Zambrano, Milano, Mimesis, 2018.

Morey, Miguel, «Las condiciones del pájaro solitario (Invitación a Ramón Gaya)», Aurora: papeles del Seminario María Zambrano, n. ${ }^{\circ}$ 5, 2003, pág. 14-21.

Ortega y Gasset, José, Pidiendo un Goethe desde dentro, en Obras completas V, Madrid, Taurus-Fundación Ortega, 2006.

Rockwell, Gray, José Ortega y Gasset, el Imperativo de la Modernidad. Una Biografia Humana e Intelectual, Madrid, Espasa Calpe, 1994. 
Trapiello, Andrés, Las armas y las letras: literatura y Guerra Civil (1936-1939), Barcelona, Austral, 2011.

Weil, Simone, La pesanteur et la grâce, Paris, Librairie Plon, 1947.

Zambrano, María, Prólogo, en Obras reunidas, Vol. 1, Madrid, Aguilar, 1971.

Zambrano, María, «A modo de autobiografía», en Anthropos, n..$^{\circ}$ 70/71, 1987, págs. 69-73.

Zambrano, María, Persona y democracia. La historia sacrificial, Barcelona, Anthropos, 1988.

Zambrano, María, Las palabras del regreso, Salamanca, Amarú Ediciones, 1995.

Zambrano, María, Los intelectuales en el drama de España y escritos de la guerra civil, Madrid, Trotta, 1998.

Zambrano, María y Laurenzi, Elena, Dante specchio umano, Troina, Città aperta, 2007.

Zambrano, María y Gaya, Ramón, Y así nos entendimos (Correspondencia 19491990), Valencia, Pre-Textos, 2018.

Zambrano, María, El idiota, Valencia, Pre-Textos, 2019. 